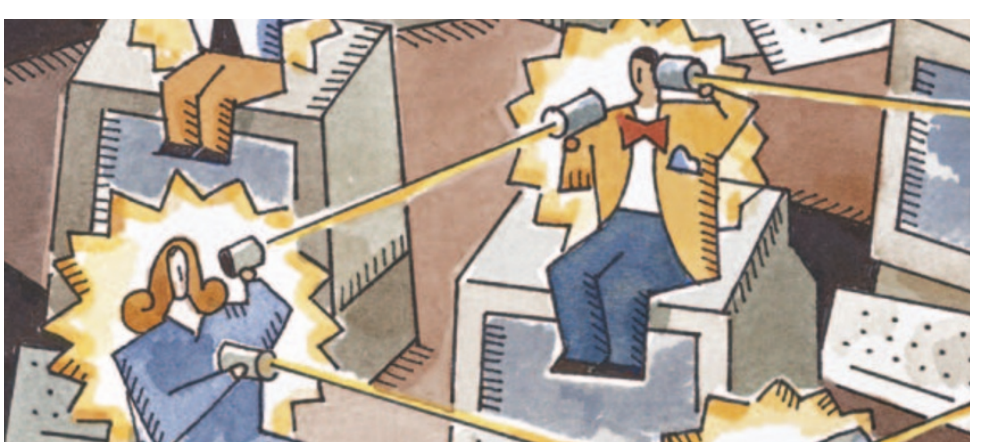

$\Rightarrow$ CELL CYCLE

\section{Signalling crosstalk}

During mitosis, duplicated chromosomes are compacted and subsequently divided into two daughter cells. Various mitotic kinases regulate these events, but how the activities of these kinases are coordinated has been unknown. Masaki Inagaki and colleagues now provide some insight into this regulatory mechanism in a report in Nature Cell Biology.

The mitotic kinase Aurora-B forms a complex with the inner centromere protein (INCENP) and survivin. INCENP is phosphorylated by Aurora-B, and Inagaki and colleagues now show that INCENP is also phosphorylated, in a mitosis-dependent manner, by cyclin-dependent kinase1 (Cdk1).

Next, Inagaki and co-workers found that a third mitotic kinase, Polo-like kinase-1 (Plk1), binds specifically to Cdk1-phosphorylated INCENP. This binding was diminished by mutating the Thr388, but not the Thr59, phosphorylation site on INCENP. The interaction between Plk1 and Thr388 phospho-INCENP was confirmed in vivo by the immunoprecipitation of INCENP from a mitotic HeLa-cell extract, as Plk1 was detected, together with Aurora-B, in the anti-INCENP precipitate.

To understand the relationship between the Aurora-B-INCENPsurvivin complex and Plk1, the authors knocked down each of the components by RNA interference. They found that the depletion of INCENP abolished the recruitment of Plk1 to kinetochores. This phenotype could be rescued by the exogenous expression of either wildtype INCENP or INCENP that was mutated at Thr59 (Thr59Ala), but not by INCENP that was mutated at Thr388 (Thr388Ala). This indicates that the phosphorylation of INCENP at Thr388 is important for the recruitment of Plk1 to kinetochores. It also implies that the role of INCENP in the localization of Plk1 is probably through direct complex formation with the kinase. In addition, the replacement of endogenous INCENP with the Thr388Ala mutant led to a delayed progression from metaphase to anaphase, thereby further confirming the biological significance of INCENP phosphorylation by Cdk1.

The authors propose that during early mitosis INCENP is phosphorylated by Aurora-B and Cdk1, and that Plk1 is recruited to Thr388 phospho-INCENP. Plk1 is thought to have an important role in spindle formation by generating the microtubule forces that pull each chromosome towards the spindle pole, whereas Aurora-B might function in ensuring the correct chromosome attachments between kinetochores and microtubules. Therefore, the signalling crosstalk among Plk1, Cdk1 and Aurora-B on INCENP, these kinases, might be a crucial step in chromosome segregation during mitosis and the progression from metaphase to anaphase.

Arianne Heinrichs

ORIGINAL RESEARCH PAPER Goto, H. et al. Complex formation of Plk1 and INCENP required for metaphase-anaphase transition. Nature Cell Biol. 8, 180-187 (2006) and the coordinated functions of
Links:

Aurora-B

http://ca.expasy.org/uniprot/O70126

INCENP

http://ca.expasy.org/uniprot/Q9WU62

Survivin

http://ca.expasy.org/uniprot/O70201

Cdk1

http://ca.expasy.org/uniprot/P11440

Plk

http://ca.expasy.org/uniprot/Q07832 